\title{
INSERT: A Novel Clinical Scanner for Simultaneous SPECT/MRI Brain Studies
}

\begin{abstract}
BF Hutton, K Erlandsson, D Salvado, M Occhipinti, Z Papp, B Tölgyesi, J Willems, C Piemonte, M Carminati, T Bukki, G Légrády, A Kuehne, Z Nyitrai, T Niendorf, P Van Mullekom, H Waiczies, K Nagy, I De Francesco, D Mathe, L Ottobrini, SC Short, C Fiorini
\end{abstract}

\begin{abstract}
A clinical SPECT insert for a commercial MRI scanner has been developed within the INSERT project, allowing simultaneous SPECT/MRI studies of the human brain. Here we present preliminary experimental results. The reconstructed resolution was 6-11 $\mathrm{mm}$ and the sensitivity $280-440 \mathrm{~s}^{-1} / \mathrm{MBq}$. The INSERT is the first clinical SPECT prototype for simultaneous SPECT/MRI.
\end{abstract}

\section{INTRODUCTION}

$\mathrm{I}^{\mathrm{N}}$ $\mathrm{N}$ recent years there has been an increasing interest in the $\mathbf{I}_{\text {development }}$ of multi-modality tomographic imaging systems, such as PET/CT [1], SPECT/CT [2] and PET/MRI [3]. A few pre-clinical SPECT/MRI systems have been developed [4-8], but no clinical system - until now [9]. The INSERT project has, for the past few years, been the working on the development of both pre-clinical and clinical SPECT inserts for commercially available MRI scanners [10]. Here we present the clinical system only.

The INSERT system is a compact stationary SPECT system. It was developed primarily for imaging brain tumours, with emphasis on sensitivity rather than resolution. Advantages of a simultaneous SPECT/MRI system include being able to use the MRI data for improving the SPECT image quality, through partial volume correction and motion correction. Also, as the system is stationary, dynamic imaging is possible. Furthermore, it is more convenient for the patients, especially those with advanced clinical symptoms, avoiding the need for two separate scans on different days.

The construction of the INSERT scanner is currently in its final stages, and we have recently been able to acquire some initial test data on the system. Previously, we also performed a series of preliminary evaluations using a single prototype detector and a rotating platform.

Manuscript received Nov 10, 2017. This work was supported by the EC FP7 grant HEALTH-305311, FCT PhD Fellowship (SFRH/BD/88093/ 2012), and NIHR University College London Hospitals Biomedical Research Centre.

BF Hutton, K Erlandsson and D Salvado are with The Inst. of Nuclear Medicine, University College London, London, UK; M Occhipinti, M Carminati and C Fiorini are with Politecnico di Milano, Dipartimento di Elettronica Informazione e Bioingegneria, Milano, Italy, and with INFN Sezione di Milano, Italy; Z Papp, B Tölgyesi, T Bukki, G Légrády, Z Nyitrai and K Nagy are with Mediso Ltd, Budapest, Hungary; J Willems and P Van Mullekom are with Nuclear Fields, Vortum-Mullem, NL; C Piemonte is with Fondazione Bruno Kessler, Trento, Italy; A Kuehne, T Niendorf, $\mathrm{H}$ Waiczies are with MRI.TOOLS GmbH, Berlin, Germany; I De Francesco is with The Dept. of Oncology, UCL Hospitals NHS Foundation Trust, London, UK; D Mathe is with CROmed Ltd, Budapest, Hungary; L Ottobrini is with The Dept. of Pathophysiology and Transplantation, University of Milan, Italy; SC Short is with The Faculty of Medicine and Health, University of Leeds, UK.

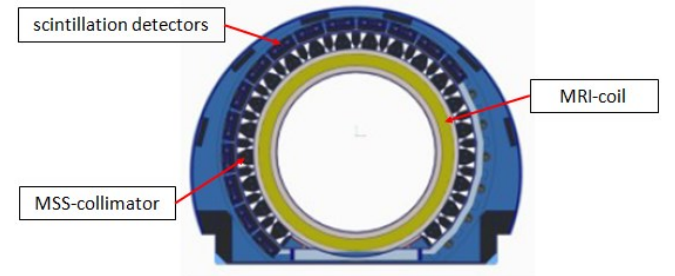

Fig. 1. Illustration of the INSERT system, showing the MRI-coil, the MSS-collimator and the scintillation detectors.

\section{MATERIALS AND METHODS}

\section{A. Scanner}

The INSERT scanner consists of a partial ring of scintillation detectors with 8-mm thick CsI(Tl)-crystals coupled to SiPM-arrays (Fig. 1). Each detector is $5 \times 10 \mathrm{~cm}^{2}$, and the partial ring contains 20 detectors. The detectors are cooled to $\sim 0^{\circ} \mathrm{C}$ with a liquid cooling system, and have an intrinsic resolution of $\sim 1 \mathrm{~mm}$ FWHM. The scanner is equipped with multi-mini-slit-slat (MSS) collimators made of Tungsten [11]. There is a dedicated transmit/receive RF-coil for MR-imaging, placed inside the ring and shielded from the readout electronics. Appropriate $\gamma$-shielding was designed based on Monte Carlo simulations [12]. Fig. 2 shows pictures of the system during assembly, with MR coil in place and with the collimator partially extracted.

\section{B. Data processing}

Data are acquired in list-mode and the positions of the detected events are determined by an ML algorithm based on estimated light-spread functions [13]. Tomographic images are reconstructed with an ML-EM or MAP-EM algorithm using a projector/back-projector based on angular blurring [14], with attenuation correction based on pseudo-CT images generated from T1- or T2-weighted MRI [15]. A practical calibration procedure was developed, based on a series of measurements for the axial and transaxial components separately [16].
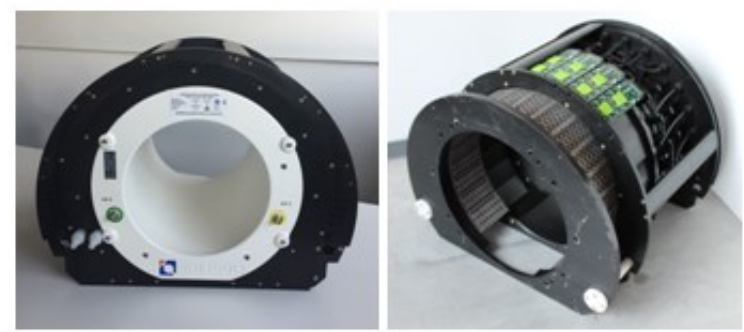

Fig. 2. INSERT system with MR coil (left), and without MR coil, showing the MSS collimator and some of the detectors (right). 


\section{Experimental evaluation}

Initially we performed a number of phantom studies with a single prototype detector $\left(5 \times 5 \mathrm{~cm}^{2}\right)$, a prototype MSS collimator and a rotating platform [16]. These studies included scanning four capillary tubes, filled with ${ }^{99 \mathrm{~m}} \mathrm{Tc}$, and placed axially at different distances from the centre of the field-ofview $(\mathrm{FoV})(25,50,75$ and $100 \mathrm{~mm})$ in air. These data were acquired for calibration purposes, but also used here for estimating the reconstructed resolution. Image reconstruction was done with 50 iterations of ML-EM. In order to avoid artificially good resolution due to the lack of background, simulated data, corresponding to a uniform background were added before, and subtracted after reconstruction. A transaxial cross-section of each source was fitted with a 2D Gaussian function, and FWHM was determined in the radial and tangential directions.

A series of experiments were also performed on the final INSERT system, starting with intrinsic and extrinsic calibration scans. Intrinsic uniformity was measured with a point source placed at the centre of the FoV. Intrinsic linearity was measured with a $5 \times 10 \mathrm{~cm}^{2}$ planar source and two different collimators with straight, parallel line apertures in the $\mathrm{x}$ - and $y$-directions, respectively. The system sensitivity variations were measured with a planar source, with the MSS collimator in place. We also scanned 4 rotating capillary sources, as described above for the single detector system, and a cylindrical phantom with a diameter of $14 \mathrm{~cm}$. We used these two scans to determine the sensitivity of the system. For the cylindrical phantom, attenuation and scatter were accounted for using a mean attenuation factor, calculated based on a reduced $\mu$-value $\left(0.1 \mathrm{~cm}^{-1}\right)$.

\section{RESULTS}

Figure 3 shows the reconstructed image of the 4 capillary sources, and also the estimated resolution in the radial and tangential directions. The resolution was $\sim 10 \mathrm{~mm}$ at the centre and, in the tangential direction, it improved towards the edge of the FoV, This trend is expected, as the source gets closer to the collimator surface. In the radial direction, on the other hand, the behaviour is less predictable. The observed pattern of increasing and decreasing resolution is something that will require further investigation.

Fig. 4 shows the $\mathrm{x}$ - and $\mathrm{y}$-linearity images for one detector, and also the system sensitivity variation map for one detector, illustrating the various mini-slit-projections obtained with the MSS collimator.

The sensitivity was estimated to $280 \mathrm{~s}^{-1} / \mathrm{MBq}$ for the rotating capillary sources, and $440 \mathrm{~s}^{-1} / \mathrm{MBq}$ for the cylindrical phantom. The large difference between these two values is probably related to the difference in the activity distribution in combination with the variation of the sensitivity over the FoV of the scanner.

\section{DISCUSSION \& CONCLUSIONS}

We have developed a SPECT insert for a commercial MRI scanner, allowing simultaneous SPECT/MRI human brain studies to be performed. Our preliminary evaluation shows that this system can provide a performance, in terms of sensitivity and resolution, at least as good as that of a standard SPECT system. Here the resolution was estimated based on $360^{\circ}$ angular sampling. Previous simulations have shown that the use of a partial ring leads to degraded resolution in the inferior part of the FoV, but also that this effect can be ameliorated by utilising anatomical MRI information during the SPECT reconstruction [17]. Future work will include further evaluations of the final system.
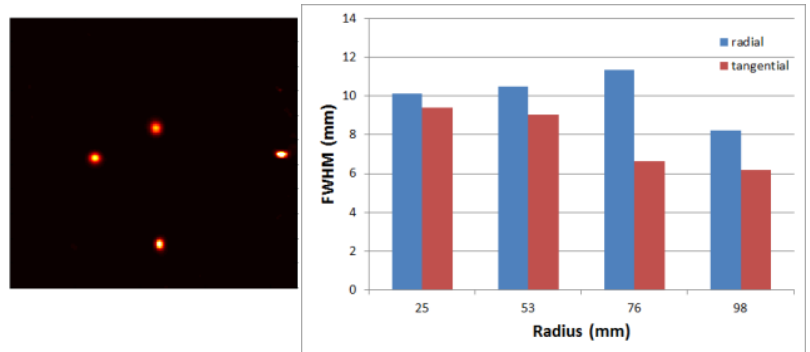

Fig. 3. Reconstructed image of 4 line sources (left), and estimated spatial resolution in the radial and tangential directions (right) for the single detector prototype system.

\section{REFERENCES}

[1] Townsend DW, Phys. Med. Biol., 53:R1-R39, 2008.

[2] Hasegawa $\mathrm{BH}$, Wong $\mathrm{KH}$, Iwata $\mathrm{K}$ et al. Technol Cancer Res Treat 2002; 1: 449-58.

[3] Pichler B. J., Kolb A., Nägele T., et al., J Nucl Med, 51:333-336, 2010.

[4] HamamuraMJ, Ha S, Roeck WW et al., Phys Med Biol; 55:1563-75, 2010.

[5] Tsui BMW, Jingyan X, Rittenbach A et al., 2011 IEEE NSS/MIC Conference Record; 2011.

[6] Van Holen R, Vandenberghe S, 2013 IEEE NSS/MIC Conf. Rec., 2013.

[7] Cai L, Lai X, Shen Z et al., Nucl. Instrum. Meth. Phys. Res. A, 734:14751, 2014.

[8] Dalm SU, Bakker IL, de Blois E, et al. J Nucl Med; 58:293-299, 2017.

[9] Hutton BF, Occhipinti M, Kuehne A, et al., Brit. J Radiol., 90:1-13, 2017

[10] Busca P, Fiorini C, Butt AD et al., Nucl. Instr. Meth. Phys. Res. A, 734(B):141-146, 2014.

[11] Salvado D, Erlandsson K, Bousse A et al., IEEE Trans. Nucl. Sci., 62:1716-1724, 2015.

[12] Salvado D, Erlandsson K, Hutton BF, 2015 IEEE NSS-MIC Conf. Rec.

[13] Occhipinti M, Busca P, Fiorini C, 2016 IEEE NSS/MICConf. Rec., 2016.

[14] Bousse A, Erlandsson K, Fuin N et al., 2013 IEEE NSS/MIC Conf. Rec.

[15] Burgos N, Cardoso MJ, Thielemans K et al., IEEE Trans. Med. Imag., 33:2332:41, 2014

[16] Salvado D, Erlandsson K, Occhipinti M et al., IEEE Trans. Radiat. Plas. Med. Sci., 2017. (under review)

[17] Erlandsson K, Salvado D, Bousse A et al., EJNMMI Physics 2015, 2 (Suppl 1):A47
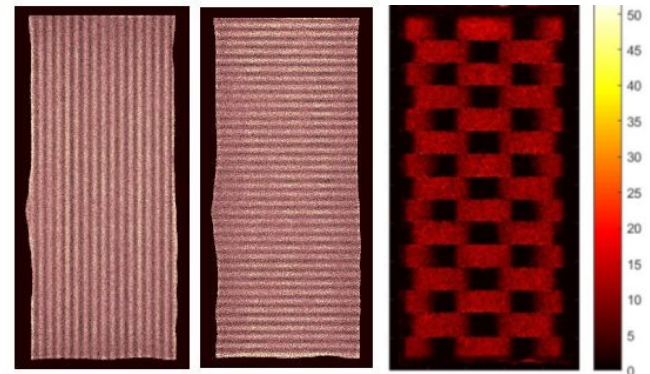

Fig. 4. Intrinsic linearity images in $x$ - and $y$-directions from the final INSERT scanner (left and centre, respectively), and system sensitivity map with MSS collimator (right). 